\title{
Paraonidae (Annelida: Polychaeta) del Parque Nacional de Coiba (Pacífico, Panamá), con la descripción de una nueva especie de Aricidea Webster, 1879
}

\author{
Paraonidae (Annelida: Polychaeta) from Coiba National Park (Pacific Ocean, Panamá), \\ with the description of a new species of Aricidea Webster, 1879
}

\section{TERESA AGUADO \& EDUARDO LÓPEZ}

\author{
Laboratorio de Biología Marina e Invertebrados, Departamento de Biología (Zoología), Facultad de Ciencias, \\ Universidad Autónoma de Madrid, Canto Blanco 28049, España; \\ e-mail: maite.aguado@uam.es
}

\begin{abstract}
RESUMEN
Durante los años 1996 y 1997 se realizó un proyecto de investigación destinado a caracterizar la fauna marina del Parque Nacional de Coiba, situado en la costa pacífica de Panamá, y en el que se prestó una atención especial a dos ambientes: los fondos arenosos sublitorales y la zona intermareal de las playas. Las muestras sublitorales se tomaron en septiembre de 1996, en dos localidades próximas a arrecifes de coral: Mali Rock y Granito de Oro. En ambos casos el sedimento estaba constituido por arena gruesa proveniente de la destrucción del coral. Las muestras intermareales se colectaron en Santa Cruz y El Gambute, dos playas asociadas a manglares en las que se seleccionaron puntos en el intermareal superior, medio e inferior. En las playas se realizaron tres muestreos, en junio de 1996, en febrero de 1997 y en noviembre de 1997. El estudio de la fauna de la familia Paraonidae (Polychaeta) reveló la presencia de nueve especies pertenecientes a tres géneros, cuatro de las cuales fueron nuevas citas para la región del Pacífico oriental comprendida entre el sur de México y norte de Colombia. Además, se describe una nueva especie de Aricidea Webster, 1879, que se caracteriza por poseer en combinación una antena extremadamente larga, que puede alcanzar el setígero 9, por el gran tamaño de los ojos del par posterior, por presentar 16 pares de branquias y por la presencia en los neuropodios abdominales de numerosas sedas pseudoarticuladas, que presentan un manubrio grueso y con la punta apenas curvada y una espina recta y de gran longitud insertada subterminalmente.
\end{abstract}

Palabras clave: Paraonidae, Aricidea, Coiba, Panamá.

\begin{abstract}
During the years 1996 and 1997, a research project aiming to characterize the marine benthic fauna of Coiba National Park, located off the Pacific coast of Panamá, was conducted and special attention was paid to two environments: sandy sublittoral bottoms and tidal flats. Sublittoral samples were taken in September of 1996, from two sites located near coral reefs: Mali Rock and Granito de Oro. In both cases, sediment was composed of coarse sand coming from coral destruction. Tidal samples were collected from Santa Cruz and El Gambute, two flats associated with mangrove systems where upper, middle and lower intertidal sampling sites were selected. Three samplings were carried out in the flats, in June of 1996, in February of 1997 and in November of 1997. The study of the Paraonidae (Polychaeta) fauna revealed the presence of nine species belonging to three different genera, four of which were new records for the Pacific area encompassed between southern Mexico and northern Colombia. Moreover, a new species of Aricidea Webster, 1879 is described; it can be distinguished from the already described species within the genus by the combined presence of an extremely long antenna, which extends to chaetiger 9, of a pair of large posterior pair eyes, of 16 pairs of branchiae, and of numerous pseudoarticulated chaetae on abdominal neuropodia. These chaetae have a thick shaft with a slightly curved tip and bear a straight and very long spine inserted subterminally.
\end{abstract}

Key words: Paraonidae, Aricidea, Coiba, Panamá. 


\section{INTRODUCCIÓN}

Los poliquetos pertenecientes a la familia $\mathrm{Pa}$ raonidae se pueden encontrar principalmente en aguas profundas de todo el mundo, aunque unas pocas especies se encuentran en aguas someras o en ambientes intermareales (Strelzov 1979). Se trata de un grupo que vive sobre el sedimento o en las capas más superficiales del mismo (Rouse \& Pleijel 2001) y al que generalmente se ha incluido en la categoría de los sedimentívoros no selectivos (Fauchald \& Jumars 1979), aunque estudios más detallados de su biología parecen indicar que al menos algunas especies son capaces de seleccionar las partículas nutritivas de entre los sedimentos (Gaston et al. 1992). La taxonomía del grupo ha sido analizada parcialmente por Hartman (1957), pero fue el trabajo de revisión de Strelzov (1979) el que sentó las bases de la actual clasificación de lo paraónidos. Actualmente se conocen alrededor de 90 especies dentro de la familia, pero el número de nuevos taxa descritos en estudios detallados sobre áreas relativamente pequeñas (Imajima 1973, Laubier \& Ramos 1973, Katzmann \& Laubier 1975) sugiere que la cifra real de especies que componen la familia puede ser mucho mayor. Por otro lado, algunos autores han comentado la necesidad de actualizar la clasificación propuesta por Strelzov (1979) a la luz de nuevos conocimientos sobre la familia (Hartley 1981).

El presente estudio fue realizado a partir de material recolectado en el Parque Nacional de Coiba durante los años 1996 y 1997 en el marco de un proyecto de investigación dirigido a caracterizar la fauna marina del mismo (San Martín et al. 1997). Este parque se sitúa en la costa pacífica de Panamá, entre los $7^{\circ} 10^{\prime}$ y los $7^{\circ} 53^{\prime} \mathrm{N}$ y entre los $81^{\circ} 32^{\prime}$ y $\operatorname{los} 81^{\circ} 56^{\prime} \mathrm{O}$, e incluye varias islas, la mayor de las cuales da nombre al mismo. Entre los ambientes estudiados en el proyecto se encontraban las playas asociadas a los manglares y los fondos blandos infralitorales, en los que se encontraron todas las especies de paraónidos mencionados en el presente trabajo.

En la isla de Coiba se localizan varios manglares con playas asociadas, dos de los cuales fueron seleccionados para el estudio: Santa Cruz y El Gambute. El primero está situado en el fondo de una pequeña ensenada en la costa NO de la isla (coordenadas $7^{\circ} 37^{\prime} 30^{\prime \prime} \mathrm{N}$, $81^{\circ} 45^{\prime} 40^{\prime \prime} \mathrm{O}$ ) y por tanto el hidrodinamismo en la playa asociada al mismo es moderado. El sedimento en toda la playa es una arena fina bastante homogénea y rica en taninos derivados de los mangles. El segundo manglar, El Gambute, está en la costa NE y próximo a la Estación Biológica de Coiba (coordenadas $7^{\circ} 37^{\prime} 25^{\prime \prime} \mathrm{N}$, $81^{\circ} 43^{\prime}$ O) y es de superficie mucho menor. En este caso, el sedimento es principalmente una mezcla de arena fangosa y grava gruesa, aunque la proporción de una y otra varía mucho de una zona a otra de la playa. Información adicional sobre las playas estudiadas en el proyecto se puede encontrar en López et al. (2002). Los fondos blandos fueron estudiados en dos localidades: Mali Rock (coordenadas $7^{\circ} 39^{\prime} \mathrm{N}$, $81^{\circ} 41^{\prime} 40^{\prime \prime}$ O) y Granito de Oro (coordenadas $7^{\circ} 35^{\prime} 30^{\prime \prime} \mathrm{N}, 81^{\circ} 42^{\prime} 30^{\prime \prime} \mathrm{O}$ ). En ambas el fondo está constituido por arena gruesa procedente de la destrucción de coral. Las localidades de muestreo se pueden observar en la Fig. 1.

\section{MATERIALES Y MÉTODOS}

La metodología empleada para la recolección de las muestras fue diferente en cada uno de los ambientes estudiados. En cada playa se tomaron muestras del intermareal superior, inferior y medio. Las playas se estudiaron en tres ocasiones, junio de 1996, noviembre de 1997 y febrero de 1997; en la primera campaña se eligió una zona de muestreo en cada playa y en las siguientes, tres (en el norte, en medio y en el sur de cada una). Cada muestra estaba formada por tres submuestras que consistieron en un cuadrado de 40 × $40 \mathrm{~cm}$ excavado hasta una profundidad de $30 \mathrm{~cm}$. El sedimento extraído fue tamizado a través de una malla de $1 \mathrm{~mm}$ de luz. Las muestras de fondos blandos submareales, recolectadas en septiembre de 1996, se obtuvieron introduciendo en el sedimento un tubo de PVC ("core") de $1 \mathrm{~L}$ de capacidad y $10 \mathrm{~cm}$ de diámetro; posteriormente fueron filtradas utilizando un tamiz de $0,1 \mathrm{~mm}$ de diámetro. Las características de las muestras (fecha de recolección, estación de muestreo y características del sedimento) se ofrecen en la Tabla 1.

El material biológico fue separado del sedimento obtenido, fijado en formol al $10 \%$ en agua de mar y posteriormente se identificaron los principales grupos taxonómicos usando la lupa binocular. En el laboratorio, los especímenes pertenecientes a la familia Paraonidae fueron estudiados utilizando tanto la lupa binocular como un microscopio óptico Nikon Optiphot provisto de sistema interferencial de contraste (Nomarsky) y cámara clara para dibujo. Los especímenes fueron identificados a nivel específico y conservados en etanol al $70 \%$, siendo depositados los tipos de la nueva especie en el Museo Nacional de Ciencias Naturales de Madrid, España, mientras el resto de los especíme- 


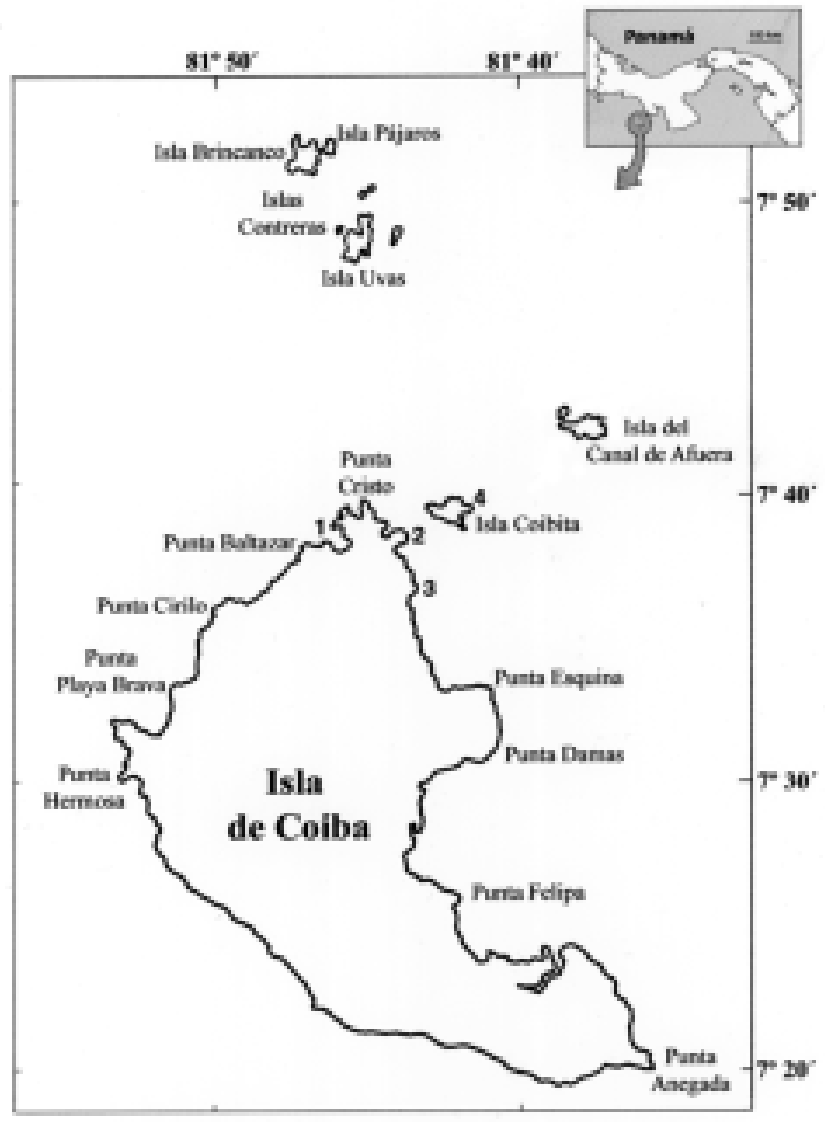

Fig. 1: Mapa de Coiba con localidades de muestreo: (1) Santa Cruz; (2) El Gambute; (3) Granito de Oro; (4) Mali Rock.

Map of Coiba with sample sites: (1) Santa Cruz; (2) El Gambute; (3) Granito de Oro; (4) Mali Rock.

TABLA 1

Características y codificación de las muestras utilizadas en el estudio

Codification and features of samples used in this study

\begin{tabular}{|c|c|c|c|c|}
\hline Muestra & Estación de muestreo & Zonación & Sedimento & Fecha \\
\hline M4C & Santa Cruz & - & Arena fina & 24/06/1996 \\
\hline M6B & Santa Cruz & - & Arena fina & 29/06/1996 \\
\hline M6C & Santa Cruz & - & Arena fina & 29/06/1996 \\
\hline AR1 & Mali Rock & Submareal - $10 \mathrm{~m}$ & Arena gruesa & $15 / 09 / 1996$ \\
\hline AR2 & Granito de Oro & Submareal $-0,5 \mathrm{~m}$ & Arena gruesa & $15 / 09 / 1996$ \\
\hline P97M1B & Santa Cruz & Intermareal medio & Arena fina & 05/02/1997 \\
\hline P97M1C & Santa Cruz & Intermareal superior & Arena fina & $05 / 02 / 1997$ \\
\hline P97M2C & Santa Cruz & Intermareal superior & Arena fangosa & $05 / 02 / 1997$ \\
\hline P97M3A & Santa Cruz & Intermareal superior & Arena fangosa & 08/02/1997 \\
\hline P97M3C & Santa Cruz & Intermareal inferior & Arena fina & 08/02/1997 \\
\hline P97M5A & El Gambute & Intermareal inferior & Arena media & $10 / 02 / 1997$ \\
\hline P97M5C & El Gambute & Intermareal superior & Arena media & $10 / 02 / 1997$ \\
\hline P97M6A & El Gambute & Intermareal inferior & Arena fangosa & $12 / 02 / 1997$ \\
\hline P97M6C & El Gambute & Intermareal superior & Arena gruesa & $12 / 02 / 1997$ \\
\hline N97M1A & El Gambute & Intermareal superior & Arena media & $14 / 11 / 1997$ \\
\hline N97M1B & El Gambute & Intermareal medio & Arena fangosa & $14 / 11 / 1997$ \\
\hline N97M1C & El Gambute & Intermareal superior & Grava & $14 / 11 / 1997$ \\
\hline N97M3C & Santa Cruz & Intermareal superior & Arena fangosa & $15 / 11 / 1997$ \\
\hline N97M5C & El Gambute & Intermareal superior & Arena gruesa & $15 / 11 / 1997$ \\
\hline N97M6A & El Gambute & Intermareal inferior & Arena media & $17 / 11 / 1997$ \\
\hline N97M6B & El Gambute & Intermareal medio & Arena fangosa & $17 / 11 / 1997$ \\
\hline N97M6C & El Gambute & Intermareal superior & Arena media & $17 / 11 / 1997$ \\
\hline
\end{tabular}


nes se encuentran depositados en la colección del Laboratorio de Biología Marina e Invertebrados de la Universidad Autónoma de Madrid.

\section{RESULTADOS}

Se identificó un total de 154 ejemplares de la familia Paraonidae (Annelida: Polychaeta) pertenecientes a tres géneros y nueve especies, una de ellas nueva para la ciencia y cuatro nuevas para la región del Pacífico oriental comprendida entre el sur de México y norte de Colombia.

\section{Género Aricidea Webster, 1879}

Aricidea (Aedicira) pacifica Hartman, 1944

Aricidea (Aedicira) pacifica: Hartman 1957: 326; Strelzov 1979: 66-68, Fig. 17(3), 25; Blake 1996: 47, Fig. 2.9.

Aricidea pacifica: Hartman 1969: 53-54, Fig. 1-3; Imajima 1973: 279-281, Fig. 14a-1.

Material examinado: P97M3A(1); N97M1A(1).

Distribución. Pacífico occidental: China, Mar Amarillo, Japón. Pacífico oriental: sur de California, Panamá (primera cita en esta área).

Aricidea (Acmira) catherinae Laubier, 1967.

Aricidea catherinae Laubier, 1967: 112-118, Figs. 4A-E, 5A-D.

Aricidea (Acesta) catherinae: Strelzov 1979: 91-93, Fig. 15(1), 38A-F.

Aricidea (Acmira) catherinae: Gaston 1984: 243 to 2-45, Fig. 2-44a-c; Blake 1996: 56-56, Fig. 2.14.

Material examinado: M6B(1).

Distribución. Atlántico noroeste: desde el Golfo de San Lorenzo hasta la Bahía de Chesapeake, Golfo de México. Mar Mediterráneo. Pacífico: California (de San Francisco hasta el sur de California), Panamá (primera cita en esta área).

Aricidea (Acmira) cerrutii pacifica Imajima, 1973.

Aricidea cerrutii pacifica Imajima, 1973: 260262, Fig. 3 a-j.

Aricidea (Acmira) cerrutii pacifica: HartmannSchröder 1991: 49-50, Fig. 81-82.

Material examinado: AR1(5); AR2(21).

Discusión. Los ejemplares de Coiba presentan algunas diferencias con las descripciones: las branquias son triangulares en vez de cirriformes, las antenas son un poco más largas en relación con el prostomio y los ganchos aparecen desplazados hacia la parte inferior del neuropodio. Estas pequeñas diferencias no se consideran relevantes, ya que el material presenta todos los caracteres diagnósticos de la especie.
Distribución. Pacífico: Japón, Australia y Panamá (primera cita en esta área).

Aricidea (Acmira) lopezi Berkeley \& Berkeley, 1956

Aricidea lopezi Berkeley \& Berkeley, 1956: 542, Fig. 1-3; Hartman 1957:318; 1969: 59, Figs. 1-3; Blake 1996: 57-59, Fig. 2.15.

Aricidea fauveli Hartman, 1957: 318; Day 1967: 560, Fig. 24.2 a-d.

Aricidea lopezi lopezi: Strelzov 1979: 102-104, Fig. 15(2), 44.

Material examinado: P97M6C(1); P97M5C(2); N97M1C(1); N97M5C(1); N97M6A(1); N97M6B(2).

Distribución. Pacífico: de Canadá hasta el sur de México, Panamá, Japón. Mar Mediterráneo. Sudáfrica.

Aricidea (Aricidea) fragilis Webster, 1879.

Aricidea fragilis: Hartman 1957: 317, pl. 43, Fig. 3; Pettibone 1965: 129-131, Fig. 1a-g, 2ae, 3a-c.

Aricidea (Aricidea) fragilis Strelzov, 1979: 6365, Fig. 21A-E.

Material examinado: P97M1B(1); P97M5A(2); P97M4A(1); N97M1B(1); P97M5B(4); N97M6A(2); N97M6B(1).

Distribución. Cosmopolita: costa este de los Estados Unidos (Virginia y sur de Carolina), Golfo de México, costa oeste de África (Angola), Mar Adriático, Mar de China (Golfo de Tonkin), Mar Amarillo (Golfo de Chihli). México (costa oeste de Baja California y Baja California sur, y Golfo de California desde Sonora a Jalisco).

Aricidea (Aricidea) wassi Pettibone, 1965.

Aricidea (Aricidea) wassi Pettibone, 1965:135138, Fig. 9-11; Strelzov 1979:62-64, Figs. 17(2), 23C-E; Katzmann \& Laubier 1975:582584, Fig.5; Blake 1996:44-45, Fig. 2.7.

Aricidea wassi Imajima, 1973:265-267, Fig.6.

Material examinado: M4C(53); M6B(24); M6C(5).

Distribución. Atlántico: de Nueva Inglaterra a Virginia, Golfo de México, Mar del Norte. Mar Adriático. Pacífico: centro y sur de California, Japón, Panamá (primera cita en este área).

Aricidea (Aricidea) sanmartini n. sp. Fig. 2. Material examinado: Holotipo: P97M2C(1), MNCN-16.01/8891. Paratipos: P97M2C(1), MNCN-16.01/8893. N97M6C(1), MNCN16.01/8892. N97M3C(1), MNCN-16.01/8894. P97M3C(2), MNCN-16.01/889. Material Adicional: P97M2C(5), MNCN-16.01/8897. P97M1C (3), MNCN-16.01/8898. 
Descripción: Holotipo (P97M2C) incompleto, con 75 setígeros, $8,7 \mathrm{~mm}$ de longitud y $0,3 \mathrm{~mm}$ de anchura a nivel del quinto setígero. Prostomio (Fig. 2A) triangular, aproximadamente igual de largo que ancho, redondeado en su parte más distal. Antena (Fig. 2A) muy larga, lisa, que parte de la mitad del prostomio y alcanza, extendida sobre el dorso, hasta el setígero 9. Dos pares de ojos (Fig. 2A) dispuestos en trapecio, los posteriores considerablemente mayores que los anteriores. Órganos nucales que se dirigen desde la parte posterior del prostomio hacia delante y rodean a los ojos posteriores, descendiendo en ángulo recto hasta la parte ventral (Fig. 2A). Labios ventrales de la boca formados por el setígero 1. Pigmentación (minúsculas manchas oscuras o pardas) en los costados de los segmentos, tanto dorsal como lateralmente. Branquias desde el setígero 4 (Fig. 2A) hasta el 20 (16 pares), aplanadas que se adelgazan hacia el extremo distal, largas (Fig. 2B, C), alcanzando más de la mitad del segmento y llegando a cruzarse en el centro de cada segmento. Segmentos medios y posteriores sin branquias (Fig. 2D). Lóbulos postsetales notopodiales muy cortos, papiliformes en los dos primeros setígeros; a partir del setígero 3 bien desarrollados; en los segmentos torácicos anteriores (Fig. 2B) son robustos, anchos y largos, hasta casi la mitad de la anchura del cuerpo, aproximadamente la mitad de largos que la branquia; en los segmentos torácicos posteriores (Fig. 2C) disminuyendo tanto de anchura como de longitud hasta estabilizarse en los segmentos abdominales (Fig. 2D) donde son claramente más delgados y más cortos, aproximadamente $2 / 3$ de la longitud de los torácicos. Sedas capilares de los segmentos prebranquiales y branquiales largas y curvadas, las neuropodiales más cortas que las notopodiales; dispuestas en varias filas (2-4), todas provistas de un borde lateral de fibrillas (Fig. 2E). En los últimos segmentos branquiales se hacen progresivamente más finas hasta transformarse en capilares rectas en los segmentos abdominales donde solo aparecen en el notopodio. Segmentos prebranquiales con 12 sedas capilares en el notopodio y 14 en el neuropodio; segmentos branquiales con menor número de sedas capilares, 6-9 en el notopodio y 1012 en el neuropodio. En el neuropodio de los segmentos 20 y 21 se mantienen dos sedas capilares y aparecen 12 sedas especiales gruesas, ligeramente curvadas en la punta y con una espina subterminal muy larga (Fig. 2F), dispuestas en dos filas. Neuropodio de los segmentos abdominales solo con sedas especiales, siendo más numerosas en el abdomen anterior que en el posterior; segmentos abdominales anteriores (del 22 al 40) con 8-10 sedas capilares en el notopodio y 16-20 sedas especiales en el neuropodio. Segmentos abdominales posteriores (del 40 en adelante) con 8-9 sedas capilares en el notopodio y 8-10 sedas especiales en el neuropodio. Pigidio desconocido.

Discusión. Aricidea (Aricidea) sanmartini n. sp. se caracteriza por poseer una antena extremadamente larga, que puede alcanzar el setígero 9, por el gran tamaño de los ojos del par posterior, por presentar 16 pares de branquias y por la presencia de numerosas sedas especiales en los neuropodios abdominales. Estas sedas pseudoarticuladas presentan un mango grueso y con la punta apenas curvada y una espina recta y de gran longitud insertada subterminalmente. Estas características se pueden observar en otras especies del género, pero nunca en combinación.

Aricidea (Allia) quadrilobata Webster \& Benedict, 1887, A. (Allia) antennata Annenkova, 1934, A. annae Laubier, 1967, y A. (Acesta) mirifica Strelzov, 1973, presentan antenas de similar longitud. Sin embargo, en estas especies las sedas especiales son claramente distintas, ya que son muy recurvadas y casi sigmoideas y carecen de espina subterminal, aunque en todas menos $A$. antennata puede aparecer una fina espina terminal. Estas especies presentan, además de en las sedas especiales, diferencias en otras estructuras. Así, A. quadrilobata, de distribución cosmopolita (Pettibone 1963, Strelzov 1979), y A. annae, del Mediterráneo (Laubier 1967), tienen un número claramente distinto de branquias (hasta 27 pares en A. quadrilobata, entre seis y diez pares en $A$. annae), en $A$. antennata, del Pacífico norte (Hartman 1969, Strelzov 1979, Blake 1996), los lóbulos notopodiales en los segmentos branquiales son por lo general bífidos, y A. mirifica, de la Antártica, pero cuya distribución podría extenderse por el Pacífico oriental hasta el golfo de California (Strelzov 1979), presenta una antena ligeramente más corta (que se extiende solo hasta el setígero 6).

Por otro lado, A. (Aricidea) minuta Southward, 1956, A. longobranquiata Day, 1961, A. (Aricidea) longicirrata HartmannSchröder, 1965, y A. (Aricidea) wassi Pettibone, 1965 , presentan sedas modificadas muy similares, pero difieren en otros aspectos de la morfología corporal: A. minuta, del Atlántico nororiental (Strelzov 1979), esta provista de una corta antena biarticulada y un prostomio claramente más largo que ancho, A longicirrata, de Chile (Hartmann-Schröder 1965, Strelzov 1979), presenta una antena muy corta (apenas de la longitud del prostomio) y branquias 


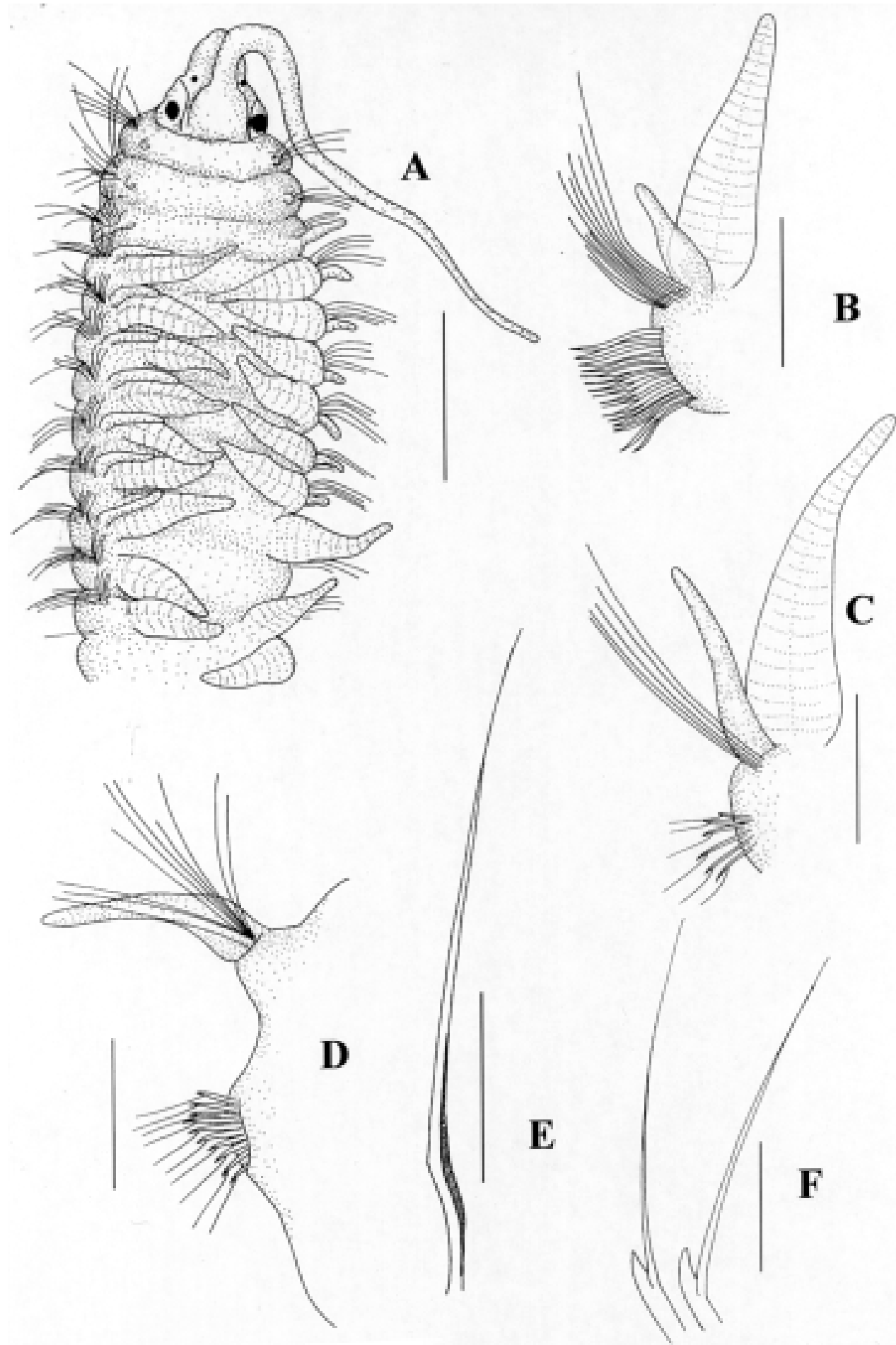

Fig. 2: Aricidea (Aricidea) sanmartini n. sp.: (A) parte anterior, vista dorsal; (B) parápodo, vista anterior, setígero 14; (C) parápodo, vista anterior, setígero 22; (D) parápodo, vista anterior, setígero 42; (E) notoseda, setígero 7; (F) neurosedas modificadas, setígero abdominal. Escala en $(\mathrm{A})=0,195$ $\mathrm{mm}$; escala en $(B-D)=97,5 \mathrm{~m}$; escala en $(E)=37 \mathrm{~m}$; escala en $(F)=20 \mathrm{~m}$.

Aricidea (Aricidea) sanmartini n. sp.: (A) anterior end, dorsal view; (B) parapodium, anterior view, chaetiger 14; (C) parapodium, anterior view, chaetiger 22; (D) parapodium, anterior view, chaetiger 42; (E) notochaeta, chaetiger 7; (F) modified neurochaetae; abdominal chaetiger. Scale in $(A)=0,195 \mathrm{~mm}$; scale in $(B-D)=97,5 \mathrm{~m}$; scale in $(E)=37 \mathrm{~m}$; scale in $(\mathrm{F})=20 \mathrm{~m}$. 
foliáceas, y A. wassi, una especie ampliamente distribuida por las regiones boreales del Pacífico y Atlántico (Pettibone 1965, Imajima 1973, Strelzov 1979, Blake 1996), tiene una antena larga pero multiarticulada y sedas modificadas con la punta algo más curvada. Aricidea longobranchiata, de África del Sur (Strelzov 1979), es la especie más similar dentro del género, siendo las únicas diferencias significativas la menor longitud de la antena, que en esta especie no sobrepasa el setígero 5, y el último par de branquias, que es más alargado.

Etimología. Esta especie está dedicada al Dr. Guillermo San Martín, de la Universidad Autónoma de Madrid, reconocido investigador en el campo de los anélidos poliquetos, coordinador y director del estudio de la fauna marina del Parque Nacional de Coiba.

\section{Género Levinsenia Mesnil, 1897}

Levinsenia gracilis (Tauber, 1879).

Paraonis (Paraonis) gracilis: Pettibone 1963:301-302, Fig.79 a-d.

Paraonis gracilis: Hartman 1957:330-331, pl.44, Figs. 4-5; 1969: 75-76, Figs. 1-3.

Paraonios gracilis gracilis: Day 1967:566, Fig. 24.4 a-b.

Tauberia gracilis: Strelzov 1979: 127-133, Figs. 14, 54-57.

Levinsenia gracilis: Blake 1996: 33-34, Fig. 2.1 .

Material examinado: P97M3C(1); N97M5C(1); N97M6A(1); N97M6B(1); M6C(1).

Distribución. Cosmopolita.

\section{Género Paradoneis Cerruti, 1909}

Paradoneis lyra Southern, 1914.

Paraonis (Paradoneis) lyra: Pettibone 1963: 300, Fig. 79 g.

Paraonides lyra: Imajima 1973: 287-288, Fig. 18 a-e; Hartman 1969: 71, Fig. 1-4.

Paradoneis lyra: Katzmann \& Laubier 1975: 569-572, Fig. 1.

Material examinado: AR2(4); N97M6B(1).

Discusión. Los ejemplares son individuos juveniles, de pequeño tamaño, con pocos pares de branquias (cinco pares) y sedas poco numerosas, que pueden llegar a faltar en los últimos segmentos posteriores. Puesto que estos caracteres están asociados a estados tempranos de desarrollo no se consideran taxonómicamente relevantes.

Distribución. Atlántico: oeste de Irlanda, Dinamarca, Sur de África, Massachusetts. Mediterráneo. Mar Negro. Pacífico: sur de California, Japón y Panamá (primera cita en esta área).

\section{AGRADECIMIENTOS}

El presente trabajo fue realizado en el marco de un proyecto de investigación financiado por la AECI (Agencia Española de Cooperación Internacional) y desarrollado con la inestimable cooperación del INRENARE panameño. Los autores expresan su gratitud hacia el personal del Parque Nacional de Coiba, así como al resto del equipo español (María Capa, Pedro Cladera, Guillermo San Martín) implicado en el proyecto.

\section{LITERATURA CITADA}

BERKELEY E \& C BERKELEY (1956) Notes on Polychaeta from the east coast of Vancouver Island and from adjacent waters, with a description of a new species of Aricidea. Journal of the Fisheries Research Board of Canada 13: 541-546.

BLAKE JA (1996) Family Paraonidae Cerruti, 1909. En: Blake JA, B Hilbig \& PH Scott (eds) The Annelida, Part 3, Polychaeta: Orbiniidae to Cossuridae, Volume 6: 27-70. Santa Bárbara Museum of Natural History, Santa Bárbara, California, USA.

DAY JH (1967) A monograph on the Polychaeta of southern Africa. Part I. Errantia; Part II. Sedentaria. Trustees of the British Museum of Natural History, London, United Kingdom. 878 pp.

FAUCHALD K \& P JUMARS (1979) The diet of worms: A study of polychaete feeding guilds. Oceanography and Marine Biology 17: 193-284.

GASTON G (1984) Family Paraonidae Cerruti, 1909. En: Uebelacker JM \& PG Johnson (eds) Taxonomic guide to the polychaetes of the northern Gulf of Mexico, Volume 1: 2.1- 2.53. Barry A. Vittor \& Associates, Inc., Mobile, Alabama, USA.

GASTON GR, JA MCLELLAND \& RW HEARD (1992) Feeding biology, distribution, and ecology of two species of benthic polychaetes: Paraonis fulgens and Paraonis pygoenigmatica (Polychaeta: Paraonidae). Gulf Research Reports 8: 395-399.

HARTLEY JP (1981) The family Paraonidae (Polychaeta) in British waters: a new species and new records with a key to species. Journal of the Marine Biological Association of the United Kingdom 61: 133-149.

HARTMAN O (1957) Orbiniidae, Apistobranchidae, Paraonidae and Longosomidae. Allan Hancock Pacific Expeditions 15: 211-393.

HARTMAN O (1969) Atlas of the Sedentariate polychaetous annelids from California. Allan Hancock Foundation, University of Southern California, Los Angeles, California, USA. 812 pp.

HARTMAN-SCHRÖDER G (1965) Zur Kenntnis des Sublittorals der chilenischen Küste unter besonderer Berücksichtigung der Polychaeten und Ostracoden. Teil 2. Die Polychaeten des Sublittorals. Mitteilungen aus dem Hamburgischen Zoologischen Museum und Institut 62: 59-305.

HARTMAN-SCHRÖDER G (1991) Zur Kenntnis des Eulitorals der australischen Küsten under besonderer Berücksichtigung der Polychaeten und Ostracoden. Teil 16. Die Polychaeten der sub und tropischen Ostküste Australiens zwische Lakes Entrance (Victoria) im Süden und Maclean (New 
South Wales) im Norden. Mitteilungen aus dem Hamburgischen Zoologischen Museum und Institut 86: 11-63.

IMAJIMA M (1973) Paraonidae (Polychaeta) from Japan. Bulletin of the National Science Museum (Japan) 16: $253-292$

KATZMANN W \& L LAUBIER (1975) Paraonidae (Polychaètes sédentaires) de l'Adriatique. Annalen der Naturhistorischen Musseum (Osterrish) 79: 567-588.

LAUBIER L (1967) Sur quelques Aricidea (Polychaeta, Paraonidae) de Banyuls-sur-Mer. Vie et Milieu A, Biologie Marine 18: 99-132.

LAUBIER L \& J RAMOS (1973) Paraonidae (Polychètes sèdentaires) de Méditerranée. Bulletin da Museum d'Histoire Naturelle (France) 113: 1097-1148.

LÓPEZ E, P CLADERA, G SAN MARTÍN, A LABORDA \& MT AGUADO (2002) Polychaete assemblages inhabiting intertidal soft bottoms associates to mangrove systems from Coiba National Park (East Pacific, Panama). Wetlands Ecology and Management 10: $233-242$.
PETTIBONE MH (1963) Marine polychaete worms of the New England region. I. Aphroditidae through Trochochaetidae. Bulletin of the United States National Museum 227: 1-356.

PETTIBONE MH (1965) Two new species of Aricidea (Polychaeta, Paraonidae) from Virginia and Florida, and redescription of Aricidea fragilis Webster. Proceedings of the Biological Society of Wahington 78: 127-140.

ROUSE GW \& F PLEIJEL (2001) Polychaeta. Oxford University Press, Oxford, United Kingdom. 354 pp.

SAN MARTÍN G, E LÓPEZ, MS REDONDO, M CAPA, P CLADERA \& A LABORDA (1997) El bentos marino del Parque Nacional de Coiba (Panamá). En: Castroviejo S (ed) Flora y fauna del Parque Nacional de Coiba (Panamá): 33-55. Agencia Española de Cooperación Internacional, Madrid, España.

STRELZOV VE (1979) Polychaete worms of the family Paraonidae Cerruti, 1909: Polychaeta Sedentaria. Akademiya Nauk SSR, San Petersburgo, República Rusa. 212 pp. 